
\title{
25 Research Soure \\ Profiling Knowledge Brokers in the Rehabilitation Sector Across Canada: A Descriptive Study
}

\section{Dina Gaid ( $\nabla$ dina.gaid@mail.mcgill.ca )}

PhD candidate Primary Affiliation: McGill University, School of Physical and Occupational Therapy Secondary Affiliation: The Centre for Interdisciplinary Research in Rehabilitation of Greater Montréal (CRIR) Address for correspondence: D39, 3654 Sir-William-Osler, Montreal, QC, Canada H3G 1Y5 https://orcid.org/0000-0003-3419-941X

\section{Sara Ahmed}

McGill University Faculty of Medicine

\section{Aliki Thomas}

McGill University Faculty of Medicine

\section{Andre Bussières}

McGill University Faculty of Medicine

\section{Research}

Keywords: Rehabilitation, knowledge brokers, knowledge brokering, knowledge translation, survey

Posted Date: September 8th, 2020

DOl: https://doi.org/10.21203/rs.3.rs-72722/v1

License: (c) (i) This work is licensed under a Creative Commons Attribution 4.0 International License. Read Full License 


\section{Abstract}

Background: Knowledge brokers (KBs) can help promote the uptake of the latest research evidence into clinical practice. Little is known about who they are, the type of work they do, and their training. Establishing a portrait of Canadian KBs working in the rehabilitation sector may inform health care organizations and knowledge translation specialists on how best to advance KBs practices. The overall goal was to describe the profile of KBs working to promote the uptake of evidence within rehabilitation settings in Canada. Specifically, this study aimed to describe the sociodemographic and professional characteristics, work activities, and training of KBs.

Methods: A cross-sectional online survey was administered to KBs working in rehabilitation settings across Canada. The survey included 20 questions covering sociodemographic and professional characteristics, work activities, and training opportunities. Response frequency and percentage were calculated for all categorical variables, and the weighted-average "WA" for each role was calculated across participants. Descriptive analysis was conducted for all open-ended questions.

Results: Of 475 participants accessing the website, 198 completed the survey questionnaire, including 99 clinicians, 35 researchers, and 26 managers. While over two-third of respondents had completed a graduate degree, only $38 \%$ reported receiving KBs-related training. The respondents' primary roles corresponded to a linking agent $(W A=1.84)$, followed by capacity builder $(W A=1.76)$, information manager (1.71), facilitator (WA=1.41), and evaluator (WA=1.32).

Conclusions: KBs are mostly expert clinicians who tend to perform brokering activities part-time targeting their peers. Participants mostly perform linking agents, capacity builder, and information roles. Moreover, few participants received formal training to perform brokering activities.

\section{Background}

Health care administrators and decision-makers emphasize the importance of evidence-based practices $(E B P)$ as a means to improve efficiency and effectiveness in service delivery. [1] However, ensuring optimal use of EBP in health-related settings remains an ongoing challenge for decision-makers and practitioners alike. [2] Knowledge translation (KT) is a process used to promote EBP in healthcare [3] and reduce the gap between routine practice and best available evidence. [4]

Several systematic reviews and meta-analyses have reported on the effectiveness of KT interventions for promoting EBP. [5-11] Overall, the use of passive KT interventions such as dissemination of printed educational materials (PEMs) [12] and professional educational conferences [9] show a 2-6\% absolute improvement in professional practice compared to no intervention. In contrast, active KT interventions, including audit and feedback [5] and educational outreach [13] have been shown to be more effective in changing professional behavior, [14] with approximately a 10\% practice change. [14-17] Several reviews suggest that the use of intermediary individuals $[18,19]$ is associated with the highest improvement in practice behavior, up to $12 \%[11,20]$ among practitioners in various healthcare disciplines, $[21-31]$ 
including rehabilitation. [14,32-38] Professionals who act as intermediaries in facilitating knowledge exchange between researchers and clinicians are referred to as human agents, [39] change agents [20] or opinion leaders, [11] while the most commonly used term in literature is brokers or knowledge brokers. [40]

Knowledge brokers (KBs) are defined as one of the human forces which bring people together to build relationships, identify practice needs, and share ideas to improve job productivity. [41] A recent systematic review reported that knowledge brokers can increase practitioners' adoption of evidencebased guidelines by 2.76 times $(95 \% \mathrm{Cl}, 2.18-3.43)$. [42] Employment of $\mathrm{KBs}$ appears to be more effective than using tailored messages alone in influencing practitioners' behavior in clinical settings. [19, 43] Thus, KT experts advocate using KBs $[44,45]$ to enhance the success and sustainability of the whole KT process, [46] and consequently reduce the research-practice gap. [41, 45, 47-53]. Since KBs activities are highly context-specific, [54, 55] their roles can vary greatly. [56] Of interest, Glegg et al. [57] recently developed the Role Model for Knowledge Brokering which encompasses all possible KBs activities that are classified into five main domains: 1) information manager, 2) linking agent, 3) capacity builder, 4) facilitator, and 5) evaluator. Additional file 1 presents each role, with definitions and examples of related tasks.

While there is evidence that KBs help reduce research-practice gap, [19, 41, 45, 47-53, 58-62] studies have found that there is a the lack of knowledge about the personal and professional characteristics of KBs in the rehabilitation context, [55] their specific work activities, $[40,63]$ and the type of training that they have received to perform their role. [41, 55, 60,64-67] This scarcity of research can limit health care organizations' ability to advance KBs practice $[45,68]$. Robust research is needed in order to 1 ) guide researchers who aim to utilize KBs in rehabilitation settings, 2) help employers to optimize the integration of KBs in rehabilitation settings, and 3) benefit KBs themselves by providing a clearer understanding of the various roles and activities they may perform to better achieve the targeted outcomes.

The overall aim of the study was to describe the profile of KBs working within rehabilitation settings in Canada. The specific objectives were to describe the sociodemographic and professional characteristics, work activities, and training of KBs.

\section{Methods}

\section{Research design}

A descriptive study design was used. We administered a cross-sectional online survey to a convenience sample of KBs working in rehabilitation institutions across Canada. The Checklist for The Checklist for Reporting Results of Internet E-Surveys (CHERRIES) is available in Additional file 2. This study was approved by the McGill University Institutional Review Board (IRB Number: A02-E11-17B). 
KBs employed with the purpose of promoting the uptake of research into clinical practice in rehabilitation institutions, whether in clinical, educational or research institutions, across Canada, were invited to participate in the study. Eligible participants: 1) were responsible for performing one or more knowledge brokering activities as an information manager, a linking agent, a capacity builder, a facilitator, or an evaluator; 2) worked full-time or part-time in any type of rehabilitation setting (e.g. hospital, rehabilitation center, professional association, research institution, academic organization); 3) could communicate in English or French; 4) had access to the internet; and 6) consented to participate.

\section{Recruitment strategies}

Three recruitment strategies were used. First, we sent recruitment emails with the information sheet, and made follow-up phone calls to all rehabilitation institutions asking them to promote the study by sending emails to their members (see Additional file 3). Second, the recruitment team made phone calls to all public hospitals and rehabilitation centres across Canada to identify KBs working in each setting. Third, a snowball strategy was used whereby KBs that were identified using the first two strategies were asked to share the recruitment email with other KBs in their network. The survey was accessible to all interested participants without password-protection. All identified KBs received a personalised e-mail invitation with a 4-minute YouTube video clarifying the five roles of KBs, and describing the study objectives and eligibility criteria as well as a hyperlink to complete a consent form prior to accessing the online survey questionnaire (see English and French videos). E-mail reminders were sent to the targeted organizations and to the identified participants up to three times within a 6 weeks period. The survey closed 4 weeks after the last reminder. The data was collected from June 2018-April 2019. Confidentiality of the data was protected by assigning each participant a unique identification number; all electronic records protected by a user password.

\section{Instrument}

The online self-administrated survey was developed through brainstorming session of the research team, which included three KT experts. Questions that aimed to identify the approaches by which the role of KBs was assigned to participants were based on the ten identifications techniques developed by Valente et al. [69] used to identify opinion leaders. As previous research has indicated that KBs and opinion leaders share mutual roles and goals, minimal adaptation was required. The Role Model for Knowledge Brokering guided questions on knowledge brokering activities. [57] The online survey consisted of 20 questions (5 pages) covering three topic areas: 1 ) socio-demographic and professional characteristics, including how the KBs role was assigned to them (10 questions [9 close-ended questions and 1 openended question]); 2) KBs' work activities (5 close-ended questions with 5-10 sub-questions each, on a 5point Likert scale from "Always" to "Never"); and 3) KBs training opportunities (5 questions [1 binary close-ended question "Yes/No" and 4 open-ended question]). Fourteen questions were mandatory to complete the survey. A professional translator translated the questions to French. Three English-speaking and three French-speaking KBs working in rehabilitation reviewed for content validity of the survey including the clarity of items, the scaling responses, the comprehensiveness of the survey, and the 
technical functionality of the electronic survey. The survey was pre-tested on the first ten KBs recruited and no additional modifications were needed. The survey was mounted on the Lime Survey platform (Version $2.63 .1+170305)$ and it took approximately $30 \mathrm{~min}$ to complete. The participants were able to review and change their answers through a Back button to review and correct their responses before submission. The Lime Survey platform provides view rates to determine the number of potential participants who logged in the website and who filled in the consent form/agreed to participate, and the PI received an email notification on every complete response (see Additional file 4).

\section{Statistical analysis}

Response frequencies and percentages were calculated for categorical variables (i.e. close-ended questions). Work activities were classified into five main roles, with a number of tasks under each role. A weighted-average (WA) was calculated for each task across participants considering response options: "always $=4$ ", "usually $=3$ ", "sometimes $=2$ ", "rarely $=1$ ", and "never $=0$ ". To obtain an overall average for each role collectively, the WA was obtained by calculating the average of all tasks for each role per participant, followed by a computation of the average of average task scores across all participants. Missing data were treated by pairwise deletion when a particular data-point was missing. Pairwise deletion is the recommended method for managing missing data as it is less biased when data is missing at random. [70]

For the five open-ended questions asking about KBs' titles and training opportunities, we used a frequency count. A deductive content analysis was conducted to categorize the qualitative data into themes. Data related to KBs professional titles were categorized into 1) health professionals', 2) knowledge translation-related, and 3) administrative. Likewise, data related to KBs training opportunities were categorized into training related to 1 ) knowledge translation and knowledge brokering, 2) research activities, 3) organizational change, 4) communication and interpersonal abilities, and 5) technology use in $\mathrm{KT}$.

\section{Results}

In total, 76 health care organizations in Canada (rehabilitation schools [ $n=19]$, regulatory bodies [ $n=20]$, professional associations [ $n=25]$, research institutions [ $n=12]$ ) were contacted by e-mail and subsequently by phone. In addition, 934 Canadian hospitals and rehabilitation centers were contacted by phone by the first author and a research assistant. Of the 475 potential participants who logged onto the survey platform, 372 agreed to participate (78\% participation rate). Although 182 participants completed all sections of the survey ( $49 \%$ completion rate), data from 198 respondents answering at least one of the knowledge brokering activities' section of the survey were included in the final analysis (Fig. 1).

Socio-demographic information

Table 1 presents the socio-demographic information of KBs. Participants were from Central Canada (71\%), Western Canada (27\%) and Eastern Canada (2\%). They spoke either English (59\%) or French 
(41\%), and the majority (73\%) was between the ages of 36-60 years old.

Table 1

Socio-demographic characteristics of

KBs $(n=198)$

\begin{tabular}{|ll|}
\hline Characteristics & $\mathbf{n}(\%)$ \\
\hline Region & \\
\hline Central & $140(71 \%)$ \\
\hline Eastern & $4(2 \%)$ \\
\hline Western & $54(27 \%)$ \\
\hline Province & \\
\hline Alberta & $20(10 \%)$ \\
\hline British Columbia & $20(10 \%)$ \\
\hline Manitoba & $13(7 \%)$ \\
\hline New Brunswick & $1(1 \%)$ \\
\hline Newfoundland & $1(1 \%)$ \\
\hline Nova Scotia & $2(1 \%)$ \\
\hline Ontario & $36(18 \%)$ \\
\hline Quebec & $104(53 \%)$ \\
\hline Saskatchewan & $1(1 \%)$ \\
\hline Language & $117(59 \%)$ \\
\hline English & $81(41 \%)$ \\
\hline French & \\
\hline Age group & $44(22 \%)$ \\
\hline Middle age (36-60) & $143 \%)$ \\
\hline Senior (> 60 years old) & $10 \%)$ \\
\hline Young age (25-35) & \\
\hline
\end{tabular}

Professional Characteristics

Table 2 presents the professional characteristics of KBs. Half of the 198 participants $(n=99)$ were clinicians, $18 \%(n=35)$ were researchers, and $13 \%(n=26)$ were managers. Of the 99 clinicians, half $(n=$ 49) were occupational therapists, $36 \%(n=36)$ were physiotherapists, $6 \%(n=6)$ were speech-language 
pathologists, and $8 \%(\mathrm{n}=8)$ reported "other" (e.g. nurses, recreational therapists). More than half $(56 \%)$ had over 15 years of clinical experience, $21 \%$ had $6-15$ years of clinical experience, and $23 \%$ had 5 years or more. The majority of participants $(n=102)$ reported their titles as health professionals (i.e., physical therapist, occupational therapist, speech-language pathologist), while fewer participants $(n=52)$ had a knowledge translation-related title (i.e., knowledge broker, knowledge translation lead, knowledge mobilization specialist, research coordinator, best practice coordinator, professional practice lead, clinician champion, clinical educator), and few had administrative titles $(n=39)$, including manager, project manager, team leader, healthcare improvement specialist, regional professional practice consultant. 
Table 2

a Professional characteristics of KBs

\begin{tabular}{|c|c|c|}
\hline Characteristics & $\mathrm{n}(\%)$ & Total \\
\hline Profession & & 198 \\
\hline Clinician & $99(50 \%)$ & \\
\hline Manager & $26(13 \%)$ & \\
\hline Researcher & $35(18 \%)$ & \\
\hline other & $62(31 \%)$ & \\
\hline Types of working organizations & & 198 \\
\hline Clinical setting & 149 (75\%) & \\
\hline Research setting & $35(18 \%)$ & \\
\hline Academic setting & $43(22 \%)$ & \\
\hline Others & $20(10 \%)$ & \\
\hline Experience in performing KBs activities & & 198 \\
\hline Beginner ( $\leq 10$ years) & $147(74 \%)$ & \\
\hline Moderate experience (11-20 years) & $40(20 \%)$ & \\
\hline Experts (Over 21 years) & $11(6 \%)$ & \\
\hline Educational level & & 198 \\
\hline Undergraduate (Diploma, Bachelor's) & $65(33 \%)$ & \\
\hline Graduate (Master, Doctoral, Post-Doc) & $133(67 \%)$ & \\
\hline Job status & & 198 \\
\hline Part-time ( $1-3$ days) & $110(56 \%)$ & \\
\hline Full-time ( $4-5$ days per week) & $88(44 \%)$ & \\
\hline Clinical professions (*) & & 99 \\
\hline Physical therapists & $36(36 \%)$ & \\
\hline Occupational therapists & $49(49 \%)$ & \\
\hline Speech language pathologists & $6(6 \%)$ & \\
\hline Others & $8(8 \%)$ & \\
\hline \multicolumn{3}{|c|}{$\left(^{*}\right)$ total number is 99 , since this is for KBs clinicians only. } \\
\hline$(* *)$ total is 110 , since this is for part-tim & only. & \\
\hline
\end{tabular}




\begin{tabular}{|c|c|c|}
\hline Characteristics & $\mathrm{n}(\%)$ & Total \\
\hline Clinical experience $(*)$ & & 99 \\
\hline Low experience ( $\leq 5$ years) & $23(23 \%)$ & \\
\hline Moderate (6-15 years) & $21(21 \%)$ & \\
\hline Expert ( $\geq 16$ years) & $55(56 \%)$ & \\
\hline Frequency of performing KBs activities (**) & & 110 \\
\hline Daily & $16(15 \%)$ & \\
\hline Weekly & $35(32 \%)$ & \\
\hline Monthly & $59(54 \%)$ & \\
\hline Payment status & & 110 \\
\hline Paid & $66(60 \%)$ & \\
\hline Non-paid & $44(40 \%)$ & \\
\hline \multicolumn{3}{|c|}{$\left.{ }^{*}\right)$ total number is 99 , since this is for KBs clinicians only. } \\
\hline$(\star \star)$ total is 110 , since this is for part-time & nly. & \\
\hline
\end{tabular}


Table 2

b Professional characteristics of KBs (*)

\begin{tabular}{|c|c|c|}
\hline Characteristics & $\mathrm{n}(\%)$ & Total $(*)$ \\
\hline Starting KBs activities & & 194 \\
\hline Volunteered & $87(45 \%)$ & \\
\hline Hired via job post & $110(57 \%)$ & \\
\hline Employers selection & $51(26 \%)$ & \\
\hline Colleagues selection & $19(10 \%)$ & \\
\hline KT expert selection & $13(7 \%)$ & \\
\hline KBs recommendation & $16(8 \%)$ & \\
\hline Evaluate abilities to perform KBs activities & $10(5 \%)$ & \\
\hline KBs networks & & 188 \\
\hline Clinician & $177(94 \%)$ & \\
\hline Researchers & $69(37 \%)$ & \\
\hline Students & $66(35 \%)$ & \\
\hline Receiving KBs training & & 182 \\
\hline Didn't receive training & $112(62 \%)$ & \\
\hline Received training & $70(38 \%)$ & \\
\hline Salary rate for full-time KBs & & 66 \\
\hline $31 \$-40 \$$ & $11(17 \%)$ & \\
\hline$\leq 30$ & $4(6 \%)$ & \\
\hline$\geq 41 \$$ & $51(77 \%)$ & \\
\hline Salary rate for part-time KBs & & 73 \\
\hline $31 \$-40 \$$ & $18(25 \%)$ & \\
\hline$\leq 30 \$$ & $5(7 \%)$ & \\
\hline$\geq 41 \$$ & $50(68 \%)$ & \\
\hline KBs perception to their salary & & 45 \\
\hline Equal to the salary of a clinician & $19(42 \%)$ & \\
\hline Less than the salary of a clinician & $9(20 \%)$ & \\
\hline
\end{tabular}




\begin{tabular}{|lcc|}
\hline Characteristics & $\mathbf{n}(\%)$ & Total $(*)$ \\
\hline More than the salary of a clinician & $17(38 \%)$ & \\
\hline$\left.{ }^{*}\right)$ Number of respondents for each question varied. \\
\hline
\end{tabular}

Seventy-five percent $(n=149)$, worked in clinical settings, $22 \%(n=43)$ in academic settings, and 18\% ( $n=$ $35)$ in research settings. Out of 188 participants, $94 \%(n=177)$ reported that they worked with clinicians, and $37 \%(n=69)$ and $35 \%(n=66)$ work with researchers and students, respectively. Regarding the frequency with which they performed their $\mathrm{KBs}$ role, more than half $(56 \%, \mathrm{n}=110)$ were part-time and, of those, $54 \%(n=59)$ were performing this role monthly, while $32 \%$ and $15 \%$ were performing their KBs role on a weekly and daily basis, respectively. Forty-four percent $(n=88)$ worked as a KBs full-time. Concerning the participants' experience as a KBs, $74 \%(n=147)$ performed this role for $\leq 10$ years, while $20 \%(n=40)$ and $6 \%(n=11)$ for $11-20$ years and over 21 years, respectively. Moreover, approaches by which the role of KBs was assigned to participants varied. Of the 194 participants, $57 \%(n=110)$ were hired following an application for a posted KBs job, while $45 \%(n=87)$ volunteered to perform this role as part of their existing position, and $26 \%$ were selected by their employers.

Seventy percent of participants (66 full-time and 73 part-time) reported on their salary. Rates were $\geq$ $41 \$ /$ hour for full-time KBs $(77 \%, n=51)$ and part-time KBs $(68 \%, n=50), 31 \$-40 \$ /$ hour $17 \%(n=11)$ of full-time KBs and $25 \%(n=18)$ of part-time, and $\leq 30 \$$ /hour for $6 \%(n=4)$ of full-time KBs, and $7 \%(n=5)$ of part-time KBs. Moreover, $19 \mathrm{KBs}$ out of $45(42 \%)$ thought that their salary was equal to the salary they would receive working as a clinician, $38 \%(n=17)$ thought that their salary was higher than clinicians', and $20 \%(n=9)$ thought that their salary was less than clinicians'.

Roles and tasks

Table 3 and Fig. 2 present the frequency of performing the KBs' five roles as well as their corresponding tasks. The primary role of participants was linking agent (weighted-average "WA" $=1.84$ ), followed by capacity builder $(W A=1.76)$, information manager $(1.71)$, facilitator $(W A=1.41)$, and evaluator $(W A=$ 1.32). 
Table 3

Activities performing the five role domains of KBs' roles and tasks

\begin{tabular}{|c|c|c|c|c|c|c|}
\hline \multirow[t]{2}{*}{ Roles (activities) } & \multirow[t]{2}{*}{ Total } & \multicolumn{3}{|c|}{ Responses n (\%) } & \multirow{2}{*}{$\begin{array}{l}\text { Task } \\
\text { weight } \\
\text { average }\end{array}$} & \multirow{2}{*}{$\begin{array}{l}\text { Role } \\
\text { weight } \\
\text { average }\end{array}$} \\
\hline & & $\begin{array}{l}\text { Usually (at } \\
\text { least } \\
\text { once/week) }\end{array}$ & $\begin{array}{l}\text { Sometimes } \\
\text { (at least } \\
\text { once/month) }\end{array}$ & $\begin{array}{l}\text { Rarely } \\
\text { (at least } \\
\text { once/ } 3 \\
\text { months) }\end{array}$ & & \\
\hline
\end{tabular}

Information Manager

Access research evidence through activities such as searching research databases journals, or research websites

Participate in self-directed learning activities such as attending webinars or workshops, or reading recent peer reviewed literature
198

$\begin{array}{llll}95(48 \%) & 60(30 \%) & 43 & 2.4 \\ & & (22 \%) & \end{array}$

$\begin{array}{llll}68(34 \%) & 82(41 \%) & 48 & 2.21 \\ & & (24 \%) & \end{array}$

Follow the latest evidence through activities such as setting up alerts for journals and reviewing them

Perform administrative activities such as organizing conferences, meetings, or workshops

Develop knowledge products such as educational material, flyers, binders, online programs, etc.

Analyze research evidence through activities such as summarizing and interpreting research results

Assess the quality of research evidence

Support applications to funding agencies

Formulate a research question (PICO question)

$\begin{array}{llll}78(39 \%) & 42(21 \%) & 78 & 1.95 \\ & & (39 \%) & \end{array}$

$57(29 \%) \quad 60(30 \%)$

$81 \quad 1.88$

(41\%)

$50(25 \%) \quad 61(31 \%)$

$87 \quad 1.8$

(44\%)

84

1.78

$48(24 \%)$

$66(33 \%)$

(42\%)

\begin{tabular}{llll}
\hline $49(25 \%)$ & $56(28 \%)$ & $\begin{array}{l}93 \\
(47 \%)\end{array}$ & 1.69 \\
\hline $19(10 \%)$ & $23(12 \%)$ & $\begin{array}{l}156 \\
(79 \%)\end{array}$ & 0.89 \\
\hline $9(5 \%)$ & $32(16 \%)$ & $\begin{array}{l}157 \\
(79 \%)\end{array}$ & 0.88 \\
\hline
\end{tabular}




\begin{tabular}{|c|c|c|c|c|c|c|}
\hline \multirow[t]{2}{*}{ Roles (activities) } & \multirow[t]{2}{*}{ Total } & \multicolumn{3}{|c|}{ Responses n (\%) } & \multirow{2}{*}{$\begin{array}{l}\text { Task } \\
\text { weight } \\
\text { average }\end{array}$} & \multirow{2}{*}{$\begin{array}{l}\text { Role } \\
\text { weight } \\
\text { average }\end{array}$} \\
\hline & & $\begin{array}{l}\text { Usually (at } \\
\text { least } \\
\text { once/week) }\end{array}$ & $\begin{array}{l}\text { Sometimes } \\
\text { (at least } \\
\text { once/month) }\end{array}$ & $\begin{array}{l}\text { Rarely } \\
\text { (at least } \\
\text { once/ } 3 \\
\text { months) }\end{array}$ & & \\
\hline $\begin{array}{l}\text { Communicate with other } \\
\text { individuals who perform } \\
\text { knowledge brokering } \\
\text { activities }\end{array}$ & & 67 (34\%) & $52(26 \%)$ & $\begin{array}{l}78 \\
(40 \%)\end{array}$ & 2.03 & 1.84 \\
\hline $\begin{array}{l}\text { Communicate with } \\
\text { stakeholders outside your } \\
\text { organization }\end{array}$ & & $65(33 \%)$ & $59(30 \%)$ & $\begin{array}{l}73 \\
(37 \%)\end{array}$ & 2.02 & \\
\hline $\begin{array}{l}\text { Identify common goals } \\
\text { among stakeholders }\end{array}$ & & 62 (31\%) & 69 (35\%) & $\begin{array}{l}66 \\
(34 \%)\end{array}$ & 1.92 & \\
\hline $\begin{array}{l}\text { Identify networking } \\
\text { opportunities for } \\
\text { stakeholders }\end{array}$ & & $53(27 \%)$ & $55(28 \%)$ & $\begin{array}{l}89 \\
(45 \%)\end{array}$ & 1.73 & \\
\hline $\begin{array}{l}\text { Develop a network or } \\
\text { community of practice }\end{array}$ & & $40(20 \%)$ & 48 (24\%) & $\begin{array}{l}109 \\
(55 \%)\end{array}$ & 1.52 & \\
\hline Capacity builder & 197 & & & & & \\
\hline $\begin{array}{l}\text { Help others apply research } \\
\text { evidence into clinical } \\
\text { practice }\end{array}$ & & 65 (33\%) & 62 (31\%) & $\begin{array}{l}70 \\
(36 \%)\end{array}$ & 1.98 & 1.76 \\
\hline $\begin{array}{l}\text { Provide relevant } \\
\text { information to your } \\
\text { stakeholders }\end{array}$ & & 64 (32\%) & 63 (32\%) & $\begin{array}{l}70 \\
(36 \%)\end{array}$ & 1.98 & \\
\hline $\begin{array}{l}\text { Design strategies to } \\
\text { address organizational } \\
\text { barriers to change the } \\
\text { practice }\end{array}$ & & $51(26 \%)$ & $66(34 \%)$ & $\begin{array}{l}80 \\
(41 \%)\end{array}$ & 1.9 & \\
\hline $\begin{array}{l}\text { Tailor resources to } \\
\text { stakeholder needs }\end{array}$ & & $56(28 \%)$ & 62 (31\%) & $\begin{array}{l}79 \\
(40 \%)\end{array}$ & 1.79 & \\
\hline $\begin{array}{l}\text { Design strategies to } \\
\text { address } \\
\text { professional/individual } \\
\text { barriers to change the } \\
\text { practice }\end{array}$ & & $62(31 \%)$ & $61(31 \%)$ & $\begin{array}{l}74 \\
(38 \%)\end{array}$ & 1.74 & \\
\hline $\begin{array}{l}\text { Tailor resources to local } \\
\text { contexts }\end{array}$ & & 58 (29\%) & $50(25 \%)$ & $\begin{array}{l}89 \\
(45 \%)\end{array}$ & 1.74 & \\
\hline $\begin{array}{l}\text { Deliver educational } \\
\text { courses, seminars, or } \\
\text { workshops to your } \\
\text { stakeholder }\end{array}$ & & $24(12 \%)$ & $56(28 \%)$ & $\begin{array}{l}117 \\
(59 \%)\end{array}$ & 1.41 & \\
\hline
\end{tabular}




\begin{tabular}{|c|c|c|c|c|c|c|}
\hline \multirow[t]{2}{*}{ Roles (activities) } & \multirow[t]{2}{*}{ Total } & \multicolumn{3}{|c|}{ Responses n (\%) } & \multirow{2}{*}{$\begin{array}{l}\text { Task } \\
\text { weight } \\
\text { average }\end{array}$} & \multirow{2}{*}{$\begin{array}{l}\text { Role } \\
\text { weight } \\
\text { average }\end{array}$} \\
\hline & & $\begin{array}{l}\text { Usually (at } \\
\text { least } \\
\text { once/week) }\end{array}$ & $\begin{array}{l}\text { Sometimes } \\
\text { (at least } \\
\text { once/month) }\end{array}$ & $\begin{array}{l}\text { Rarely } \\
\text { (at least } \\
\text { once/ } 3 \\
\text { months) }\end{array}$ & & \\
\hline Facilitator & 190 & & & & & \\
\hline $\begin{array}{l}\text { Promote knowledge } \\
\text { exchange among } \\
\text { stakeholders (e.g. by } \\
\text { supporting peer-to-peer } \\
\text { learning) }\end{array}$ & & $54(28 \%)$ & $69(36 \%)$ & $\begin{array}{l}67 \\
(35 \%)\end{array}$ & 1.96 & 1.41 \\
\hline $\begin{array}{l}\text { Facilitate workshops, } \\
\text { follow-up sessions, } \\
\text { individual and group } \\
\text { discussions }\end{array}$ & & $43(23 \%)$ & $65(34 \%)$ & $\begin{array}{l}82 \\
(43 \%)\end{array}$ & 1.82 & \\
\hline $\begin{array}{l}\text { Facilitate organizational } \\
\text { changes }\end{array}$ & & $38(20 \%)$ & $60(32 \%)$ & $\begin{array}{l}92 \\
(48 \%)\end{array}$ & 1.64 & \\
\hline $\begin{array}{l}\text { Promote reflective practice } \\
\text { such as leading } \\
\text { discussion groups or/and } \\
\text { peer review activities } \\
\text { touching on clinical } \\
\text { practices }\end{array}$ & & $32(17 \%)$ & $63(33 \%)$ & $\begin{array}{l}95 \\
(50 \%)\end{array}$ & 1.55 & \\
\hline $\begin{array}{l}\text { Guide ongoing } \\
\text { collaborative learning }\end{array}$ & & $30(16 \%)$ & $44(23 \%)$ & $\begin{array}{l}116 \\
(61 \%)\end{array}$ & 1.25 & \\
\hline $\begin{array}{l}\text { Organize schedules to } \\
\text { hold educational meetings } \\
\text { during the workday }\end{array}$ & & $23(12 \%)$ & $52(27 \%)$ & $\begin{array}{l}115 \\
(61 \%)\end{array}$ & 1.22 & \\
\hline $\begin{array}{l}\text { Facilitate online } \\
\text { discussion boards }\end{array}$ & & $6(3 \%)$ & $15(8 \%)$ & $\begin{array}{l}169 \\
(89 \%)\end{array}$ & 0.45 & \\
\hline Evaluator & 190 & & & & & \\
\hline $\begin{array}{l}\text { Evaluate the impact of } \\
\text { your knowledge brokering } \\
\text { activities }\end{array}$ & & $14(7 \%)$ & $42(22 \%)$ & $\begin{array}{l}134 \\
(71 \%)\end{array}$ & 2.21 & 1.32 \\
\hline $\begin{array}{l}\text { Identify opportunities for } \\
\text { integrating evidence into } \\
\text { practice }\end{array}$ & & 55 (29\%) & 65 (34\%) & $\begin{array}{l}70 \\
(37 \%)\end{array}$ & 1.91 & \\
\hline $\begin{array}{l}\text { Identify relevant } \\
\text { stakeholders }\end{array}$ & & $54(28 \%)$ & 60 (32\%) & $\begin{array}{l}76 \\
(40 \%)\end{array}$ & 1.82 & \\
\hline $\begin{array}{l}\text { Assess professional } \\
\text { barriers to practice change } \\
\text { and/or practice change } \\
\text { facilitators }\end{array}$ & & 29 (15\%) & 66 (35\%) & $\begin{array}{l}95 \\
(50 \%)\end{array}$ & 1.52 & \\
\hline
\end{tabular}




\begin{tabular}{|lllll|}
\hline Roles (activities) & Total & \multicolumn{2}{c}{ Responses n (\%) } & \multicolumn{2}{l|}{$\begin{array}{l}\text { Task } \\
\text { weight } \\
\text { average }\end{array}$} & $\begin{array}{l}\text { Role } \\
\text { weight } \\
\text { average }\end{array}$ \\
\cline { 2 - 5 } & $\begin{array}{l}\text { Usually (at } \\
\text { least } \\
\text { once/week) }\end{array}$ & $\begin{array}{l}\text { Sometimes } \\
\text { (at least } \\
\text { once/month) }\end{array}$ & $\begin{array}{l}\text { Rarely } \\
\text { (at least } \\
\text { once/ } \\
\text { months) }\end{array}$ & \\
\hline $\begin{array}{l}\text { Assess organizational } \\
\text { barriers to practice change } \\
\text { and/or practice change } \\
\text { facilitators }\end{array}$ & $31(16 \%)$ & $61(32 \%)$ & $\begin{array}{l}98 \\
(52 \%)\end{array}$ & 1.51 \\
\hline $\begin{array}{l}\text { Conduct environmental } \\
\text { scans or needs } \\
\text { assessments }\end{array}$ & $21(11 \%)$ & $53(28 \%)$ & $\begin{array}{l}116 \\
(61 \%)\end{array}$ & 1.36 \\
\hline $\begin{array}{l}\text { Assess organizational } \\
\text { capacity for change }\end{array}$ & $20(11 \%)$ & $49(26 \%)$ & $\begin{array}{l}121 \\
(64 \%)\end{array}$ & 1.17 \\
\hline $\begin{array}{l}\text { Evaluate KT and/or } \\
\text { implementation process } \\
\text { outcomes }\end{array}$ & $19(10 \%)$ & $40(21 \%)$ & $\begin{array}{l}131 \\
(69 \%)\end{array}$ & 1.13 \\
\hline $\begin{array}{l}\text { Integrate KT frameworks } \\
\text { and evidence into } \\
\text { evaluation processes }\end{array}$ & $25(13 \%)$ & $30(16 \%)$ & $\begin{array}{l}135 \\
(71 \%)\end{array}$ & 1.06 \\
\hline $\begin{array}{l}\text { Evaluate linkage and } \\
\text { exchange networks }\end{array}$ & $7(4 \%)$ & $28(15 \%)$ & $\begin{array}{l}155 \\
(82 \%)\end{array}$ & 0.71 \\
\hline
\end{tabular}

The most common tasks of the linking agent role were "Communicating with other individuals who perform knowledge brokering activities" (WA $=2.03$ ) and "Communicating with stakeholders outside your organization" (WA =2.02), followed by "Identifying common goals among stakeholders" (WA = 1.92). For the capacity builder role, the most common tasks were "Helping others apply research evidence into clinical practice" (WA =1.98), "Providing relevant information to your stakeholders" (WA $=1.98$ ), and "Design strategies to address organizational barriers to change the practice" (WA $=1.9$ ). For the information manager role, the most common tasks were "Access research evidence through activities such as searching research databases journals, or research websites" (WA = 2.4), "Participate in selfdirected learning activities such as attending webinars or workshops, or reading recent peer-reviewed literature" (WA = 2.21), "Follow the latest evidence through activities such as setting up alerts for journals and reviewing them" (WA =1.95), "Perform administrative activities such as organizing conferences, meetings, or workshops" (WA =1.88), and "Develop knowledge products such as educational material, flyers, binders, online programs" (WA =1.8), respectively. With respect to facilitator role, the most common tasks were "Promote knowledge exchange among stakeholders (e.g. by supporting peer-to-peer learning)" (WA =1.96), and "Facilitate workshops, follow-up sessions, individual and group discussions" (WA = 1.82). Concerning the evaluator role, the most common tasks were "Evaluate the impact of your knowledge brokering activities (WA $=2.21$ ), "Identify opportunities for integrating evidence into practice" $(W A=1.91)$, and "Identify relevant stakeholders" (WA =1.82). 
Training opportunities

Of the 198 participants, $67 \%(n=133)$ had completed graduate studies (e.g., master's, doctoral or postdoctoral degree). Only $38 \%(n=70)$ indicated having received some training to undertake or perform their $\mathrm{KBs}$ role. Training covered a variety of topics including knowledge translation and knowledge brokering (i.e., knowledge translation professional certificate, practicing knowledge translation, knowledge mobilization certificate), research activities (i.e., program evaluation), and communication abilities (i.e., leadership, emotional intelligence, and coaching). The most common approaches through which participants were informed about training opportunities included suggestions from a colleague/employer/manager $(n=25)$, through online searches $(n=22)$, and via newsletter subscriptions $(n=19)$. Many participants $(n=115)$ indicated needing additional training to be able to fulfill their roles. Proposed topics included knowledge translation, knowledge brokering, research topics (i.e., searching, assessing, and synthesizing evidence), organizational change strategies, communication and interpersonal abilities, and lastly skills for using technology in KT (i.e.; mobile applications and video games).

\section{Discussion}

This study provides new insights on KBs' demographic and professional characteristics, work activities in the rehabilitation setting, and training opportunities. Our results indicate that a large proportion of KBs are experienced clinicians, reporting over 15 years of clinical work. Most perform their brokering activities part-time. These findings are consistent with a realist review by our team, showing that individuals who perform knowledge brokering activities are clinicians embedded within rehabilitation settings. This is also consistent with evidence from other healthcare sectors recommending that KBs be clinicians working in clinical settings, $[19,71]$ as experienced clinicians having a dual role as KBs may be more aware of their peers' needs, current practices, various clinical roles, busy schedules and caseloads. [1, 58, 72] Such extensive knowledge of their environment may help KBs tailor research evidence to working clinicians.

As in previous research, the KBs role in this study was neither official nor was it explicit. [40] Our findings showed that several titles are used to 'label' individuals performing KBs roles (e.g., physiotherapist, occupational therapist, knowledge mobilization specialist, research coordinator, professional practice lead, clinician champion, clinical educator), as knowledge brokering activities are usually embedded within the function of managers, opinion leaders, researchers, and educators. $[41,52,73,74]$ The breadth of health professionals and employment titles of KBs suggests that the KBs roles are supported by a range of individuals that incorporate knowledge brokering within their role.

Personal attributes of KBs included being positive, persuasive, entrepreneurial, proactive, enthusiastic, and self-motivated. [75] Highly motivated and enthusiastic KBs can positively influence various stakeholder groups. $[1,72]$ Our findings tend to support these features by showing that many KBs were proactive and self-motivated to the extent that they were willing to perform the brokering role on a voluntary basis. 
Previous research has reported the main roles for KBs as linkage and exchange, [76-78] capacitybuilding, $[3,19,66,79-81]$ and knowledge management. $[45,76,79]$ Similarly, our findings showed that the most frequent roles for KBs were linking agents, followed by capacity builder, and information manager. Specifically, at the task level, our results suggested that KBs tended to more often perform selfdirected learning tasks (e.g., search for research evidence, evaluate the impact of brokering activities) compared to tasks requiring engagement with other individuals (e.g., communicating with others KBs and with stakeholders outside their organization). Self-directed learning tasks may be easier to accomplish as these depend on their own time, initiative and motivation, while the tasks requiring engagement with other individuals may be influenced by the organizational culture and constraints, which can reduce end-users' tendency to be engaged in KBs activities. This supports the need for valuing and prioritising brokering roles and activities within the organization to increase the likelihood of professional behaviour change. [40] More time-consuming tasks (e.g., develop knowledge products such as educational material, facilitate workshops and group discussions, identify relevant stakeholders, and administrative activities such as organizing conferences) were less frequent, possibly because part-time KBs have to prioritize tasks. Lack of time appears to be a constant barrier for clinicians who perform brokering activities, [40] having to handle competing priorities and managing various tasks while meeting the responsibilities of their roles. It is possible that employers may need to liberate KBs clinical schedules so they can undertake important time-consuming activities. Another suggestion may be for KBs to collaborate with researchers and graduate students to help with some of the research-related tasks.

Although very few participants received formal training to perform brokering activities, most had higher education credentials (e.g. Master's degree) and had performed the KBs role for over 10 years. As KBs activities are context-specific, [54] it seems that performing similar types of activities that address local needs for long periods of time may help KBs cumulate experience in performing the KBs role and compensate the lack of formal KBs training. Nonetheless, most participants highlighted a need for additional training to increase their knowledge and skills in several areas such as knowledge translation, knowledge brokering, research topics, organizational change strategies, communication and interpersonal abilities. The diversity of training topics identified reflects the wide range of KBs roles and highlights that brokering activities are highly responsive to the real-world environments in which KBs work. [54] Thus, training opportunities should not simply focus on exploring effective KT strategies, but they should aim to target all competencies and roles that KBs are expected to fulfill, including research skills, barriers to and strategies for organizational change, and communication skills. In addition, training of Canadian KBs should be available in both English and French, as the majority were located in central Canada (Ontario and Quebec), and advertised through diverse medium (e.g., colleagues, online announcements, newsletters of professional organisations).

Identifying the financial compensation of KBs was an important finding from this research that can serve to stimulate conversations with future employers. The majority of KBs reported receiving an hourly rate that is equal to their salary as clinicians. Taking into consideration that KBs are expert clinicians performing activities aimed at improving overall clinical practice to improve patient health, they should be compensated fairly. Previous research indicated that dedicating financial support for research activities 
can facilitate brokering activities. [40] In part, additional financial support for KBs may be possible by encouraging collaborations between KBs and researchers in order to take advantage of funded research investigating brokering activities. [40] However, it is important for research projects to consider early on the sustainability of the KBs role for organisations to plan as needed to maintain the KBs role in the longterm.

A number of studies are still needed to increase our knowledge about KBs and improve KBs impact on rehabilitation practices. First, there is a need to explore the relationships between KBs demographics, professional characteristics, and work-related activities with successful implementation of the KBs in specific contexts to better guide researchers, employers and managers when identifying KBs hired to perform particular roles. Second, research that explores the types, characteristic, and content of available training opportunities for KBs could help address some of their training needs. Lastly, we need to explore the barriers and the facilitators that KBs face during the brokering process.

\section{Strengths And Limitation}

A major strength of this research was the use of three recruitment strategies covering numerous academic, research, and clinical institutions across Canada as well as professional and regulatory bodies. A response rate however cannot be calculated, as the denominator is unknown. Second, using an online self-administrated survey was efficient in saving time and cost, and helped to administer the survey across Canada. Third, while previous researchers have discussed the roles and activities performed by KBs in healthcare $[57,82]$ this study is the first study reporting on KBs' roles and activities by surveying a large number of KBs in rehabilitation across Canada, thereby increasing the generalizability of our findings. Nonetheless, this study has some limitations. First, individuals decided to participate in the survey based on self-identification. To address this limitation, we clarified the characteristics of the targeted participants through a short YouTube video attached to the invitation emails. Second, there were challenges with circulating the invitation email among members of some academic institutions due to local constraints related to research ethics, which meant that some organisations could not be included in the recruitment strategy. Third, the lower response rate to sensitive survey questions (e.g. respondents' salary) may limit the generalizability of the related results. Fourth, our results cannot be generalised to private clinical settings, as it was difficult to target all rehabilitation settings (public and private) due to limited resources and time constraints. Lastly, the survey instrument was only validated for face and content validity. The work activities section was guided by the Role Model for Knowledge Brokering; however, more items can be generated for each role in the future to ensure covering all possible tasks in each KBs role. The items selected in this study were those perceived to be most reflective of a given role, and the total number of items included was based on maintaining a balance between content coverage and the time needed to complete the survey.

\section{Conclusions}


This research is an important first step in exploring the profile of KBs working to promote the uptake of evidence within rehabilitation contexts in Canada. Findings suggest that KBs are mostly expert clinicians who tend to perform brokering activities targeting their peers on a part-time basis and as part of their health professional position. Several titles are used interchangeably to refer to KBs, who mostly performed the role of linking agent, capacity builder, and information manager. Moreover, few participants received any formal training in performing brokering activities. There is a need to explore the available training opportunities for KBs to help in addressing their training needs. These findings provide valuable information to organisations wishing to employ KBs to help improve clinical practice, and ultimately patient health outcomes.

\section{List Of Abbreviations}

KT

Knowledge translation

KBs

Knowledge brokers

CHERRIES

The Checklist for Reporting Results of Internet E-Surveys

WA

Weighted-Average

\section{Declarations}

\section{Ethics approval and consent to participate}

This study was approved by the McGill University Institutional Review Board (IRB Number: A02-E11-17B). All research participants provided written consent before completing the survey.

\section{Consent for publication}

The consent to participate in the study included all participants providing consent for publication in an unidentifiable format.

\section{Availability of data and materials}

The datasets used and/or analysed during the current study are available from the corresponding author on reasonable request.

\section{Competing interests}


The authors declare that they have no competing interests.

\section{Funding}

No funding was sought for this study.

\section{Authors' contributions}

DG developed data collection tool, collected, prepared data, coded, analysed and interpreted data. SA, AT, and $A B$ contributed to the conceptualisation of the manuscript and critically reviewed the manuscript. All authors read and approved the final manuscript.

\section{Acknowledgements}

We wish to thank Miss Mushirah Hossenbaccus, research assistant, Le Centre intégré universitaire de santé et de services sociaux du Centre-Ouest-de-l'île-de-Montréal, The Centre for Interdisciplinary Research in Rehabilitation of Greater Montréal (CRIR) for her assistance with making phone calls for clinical sites in Quebec, and Mr. Ahmed Abou-Sharkh, PhD student, for recording the French version of the YouTube video, and McGill IT services for their support with the Lime Survey platform hosted by McGill University.

\section{References}

1. Cameron D, Russell DJ, Rivard L, Darrah J, Palisano R. Knowledge brokering in children's rehabilitation organizations: perspectives from administrators. J Contin Educ Health Prof. 2011;31(1):28-33.

2. Straus SE, Tetroe J, Graham I. Defining knowledge translation. CMAJ. 2009;181(3-4):165-8.

3. Traynor R, DeCorby K, Dobbins M. Knowledge brokering in public health: a tale of two studies. Public Health. 2014;128(6):533-44.

4. Grimshaw JM, Eccles MP, Lavis JN, Hill SJ, Squires JE. Knowledge translation of research findings. Implement Sci. 2012;7:50.

5. Jamtvedt G, Young JM, Kristoffersen DT, O'Brien MA, Oxman AD. Audit and feedback: effects on professional practice and health care outcomes. Cochrane Database Syst Rev. 2006;(2):CD000259.

6. Grimshaw JM, Thomas RE, MacLennan G, et al. Effectiveness and efficiency of guideline dissemination and implementation strategies. Health Technol Assess. 2004;8(6):iii-72.

7. French SD, Green S, Buchbinder R, Barnes H. Interventions for improving the appropriate use of imaging in people with musculoskeletal conditions. Cochrane Database Syst Rev. 2010;2010(1):CD006094.

8. Farmer AP, Légaré $F$, Turcot $L$, et al. Printed educational materials: effects on professional practice and health care outcomes. Cochrane Database Syst Rev. 2008;(3):CD004398. 
9. Forsetlund L, Bjørndal A, Rashidian A, et al. Continuing education meetings and workshops: effects on professional practice and health care outcomes. Cochrane Database Syst Rev. 2009;2009(2):CD003030.

10. Hakkennes S, Dodd K. Guideline implementation in allied health professions: a systematic review of the literature. Qual Saf Health Care. 2008;17(4):296-300.

11. Flodgren G, Parmelli E, Doumit G, et al. Local opinion leaders: effects on professional practice and health care outcomes. Cochrane Database Syst Rev. 2011;(8):CD000125.

12. Giguère $A$, Légaré $F$, Grimshaw $J$, et al. Printed educational materials: effects on professional practice and healthcare outcomes. Cochrane Database Syst Rev. 2012;10(10):CD004398.

13. O'Brien MA, Rogers S, Jamtvedt G, et al. Educational outreach visits: effects on professional practice and health care outcomes. Cochrane Database Syst Rev. 2007;2007(4):CD000409.

14. Rebbeck T, Macedo LG, Maher CG. Compliance with clinical guidelines for whiplash improved with a targeted implementation strategy: a prospective cohort study. BMC Health Serv Res. 2013;13:213.

15. Armstrong $\mathrm{R}$, Waters $\mathrm{E}$, Crockett $\mathrm{B}$, Keleher $\mathrm{H}$. The nature of evidence resources and knowledge translation for health promotion practitioners. Health Promot Int. 2007;22(3):254-60.

16. Grimshaw JM, Shirran L, Thomas R, et al. Changing provider behavior: an overview of systematic reviews of interventions. Med Care. 2001;39(8 Suppl 2):II2-45.

17. Grimshaw J, Eccles M, Thomas R, et al. Toward evidence-based quality improvement. Evidence (and its limitations) of the effectiveness of guideline dissemination and implementation strategies 19661998. J Gen Intern Med. 2006;21(Suppl 2(Suppl 2):14-20.

18. Doumit G, Gattellari M, Grimshaw J, O'Brien MA. Local opinion leaders: effects on professional practice and health care outcomes. Cochrane Database Syst Rev. 2007;(1):CD000125.

19. Elueze IN. Evaluating the effectiveness of knowledge brokering in health research: a systematised review with some bibliometric information. Health Info Libr J. 2015;32(3):168-81.

20. McCormack B, Rycroft-Malone J, Decorby K, et al. A realist review of interventions and strategies to promote evidence-informed healthcare: a focus on change agency. Implement Sci. 2013;8:107.

21. Rogers EM. Lessons for guidelines from the diffusion of innovations. Jt Comm J Qual Improv. 1995;21(7):324-8.

22. Gifford DR, Holloway RG, Frankel MR, et al. Improving adherence to dementia guidelines through education and opinion leaders. A randomized, controlled trial. Ann Intern Med. 1999;131(4):237-46.

23. Ryan DP, Marlow B, Fisher R. Educationally influential physicians: the need for construct validation. J Contin Educ Health Prof. 2002;22(3):160-9.

24. Yancey AK, Siegel JM, McDaniel KL. Role models, ethnic identity, and health-risk behaviors in urban adolescents. Arch Pediatr Adolesc Med. 2002;156(1):55-61.

25. Earp JA, Eng E, O'Malley MS, et al. Increasing use of mammography among older, rural African American women: results from a community trial. Am J Public Health. 2002;92(4):646-54. 
26. Valente TW, Hoffman BR, Ritt-Olson A, Lichtman K, Johnson CA. Effects of a social-network method for group assignment strategies on peer-led tobacco prevention programs in schools. Am J Public Health. 2003;93(11):1837-43.

27. Perry CL, Komro KA, Veblen-Mortenson S, et al. A randomized controlled trial of the middle and junior high school D.A.R.E. and D.A.R.E. Plus programs. Arch Pediatr Adolesc Med. 2003;157(2):178-84.

28. Ellerbeck E. Opinion leaders vs audit feedback to implement practice guidelines. JAMA. 1991;266(9):1217.

29. Kelly JA, St Lawrence JS, Diaz YE, et al. HIV risk behavior reduction following intervention with key opinion leaders of population: an experimental analysis. Am J Public Health. 1991;81(2):168-71.

30. Latkin CA. Outreach in natural settings: the use of peer leaders for HIV prevention among injecting drug users' networks. Public Health Rep. 1998;113(Suppl 1(Suppl 1):151-9.

31. Sikkema KJ, Kelly JA, Winett RA, et al. Outcomes of a randomized community-level HIV prevention intervention for women living in 18 low-income housing developments. Am J Public Health. 2000;90(1):57-63.

32. Ammendolia C, Hogg-Johnson S, Pennick V, Glazier R, Bombardier C. Implementing evidence-based guidelines for radiography in acute low back pain: a pilot study in a chiropractic community. $J$ Manipulative Physiol Ther. 2004;27(3):170-9.

33. Stevenson K, Lewis M, Hay E. Do physiotherapists' attitudes towards evidence-based practice change as a result of an evidence-based educational programme? J Eval Clin Pract. 2004;10(2):207-17.

34. Stevenson K, Lewis M, Hay E. Does physiotherapy management of low back pain change as a result of an evidence-based educational programme? J Eval Clin Pract. 2006;12(3):365-75.

35. Rebbeck T, Maher CG, Refshauge KM. Evaluating two implementation strategies for whiplash guidelines in physiotherapy: a cluster randomised trial. Aust J Physiother. 2006;52(3):165-74.

36. Gross DP, Lowe A. Evaluation of a knowledge translation initiative for physical therapists treating patients with work disability. Disabil Rehabil. 2009;31(11):871-9.

37. Karlen E, McCathie B. Implementation of a Quality Improvement Process Aimed to Deliver HigherValue Physical Therapy for Patients With Low Back Pain: Case Report. Phys Ther. 2015;95(12):1712-21.

38. Lynch EA, Cadilhac DA, Luker JA, Hillier SL. Education-only versus a multifaceted intervention for improving assessment of rehabilitation needs after stroke; a cluster randomised trial. Implement Sci. 2016;11(1):120.

39. David Johnson J. The role of human agents in facilitating clinical and translational science. Clin Transl Sci. 2012;5(4):356-61.

40. Bartelink ME, Baggen Y, Stevens DE, et al. Facilitators and barriers to brokering between research and care by senior clinical-scientists in general practice and elderly care medicine. Educ Prim Care. 2019;30(2):80-7. 
41. CHSRF, The Theory and Practice of Knowledge Brokering in Canada's Health System: A Report Based on a CHSRF National Consultation and a Literature Review December 2003. 2003: Canadian Health Services Research Foundation. Available at:

https://books.google.ca/books/about/The_Theory_and_Practice_of_Knowledge_Bro.html? id=GR3VNwAACAAJ\&redir_esc=y.

42. Baskerville NB, Liddy C, Hogg W. Systematic review and meta-analysis of practice facilitation within primary care settings. Ann Fam Med. 2012;10(1):63-74.

43. Dobbins M, DeCorby K, Robeson P, Ciliska D, Thomas H, Hanna S. The power of tailored messaging: preliminary results from Canada's first trial on knowledge brokering. Ottawa: Canadian Cochrane Colloquium. 2007;5.

44. Dobbins M, DeCorby K, Twiddy T. A knowledge transfer strategy for public health decision makers. Worldviews Evid Based Nurs. 2004;1(2):120-8.

45. Ward V, House A, Hamer S. Knowledge Brokering: The missing link in the evidence to action chain? Evid Policy. 2009;5(3):267-79.

46. Zidarov D, Thomas A, Poissant L. Knowledge translation in physical therapy: from theory to practice. Disabil Rehabil. 2013;35(18):1571-7.

47. King G, Wright V, Russell DJ. Understanding paediatric rehabilitation therapists' lack of use of outcome measures. Disabil Rehabil. 2011;33(25-26):2662-71.

48. Ward VL, House AO, Hamer S. Knowledge brokering: exploring the process of transferring knowledge into action. BMC Health Serv Res. 2009;9:12.

49. Heng HK, McGeorge WD, Loosemore M. Beyond strategy: exploring the brokerage role of facilities manager in hospitals. J Health Organ Manag. 2005;19(1):16-31.

50. Wahabi HA, Al-Ansary LA. Innovative teaching methods for capacity building in knowledge translation. BMC Med Educ. 2011;11:85.

51. Urquhart R, Porter GA, Grunfeld E. Reflections on knowledge brokering within a multidisciplinary research team. J Contin Educ Health Prof. 2011;31(4):283-90.

52. Dobbins M, Hanna SE, Ciliska D, et al. A randomized controlled trial evaluating the impact of knowledge translation and exchange strategies. Implement Sci. 2009;4:61.

53. Tran NT, Hyder AA, Kulanthayan S, Singh S, Umar RS. Engaging policy makers in road safety research in Malaysia: a theoretical and contextual analysis. Health Policy. 2009;90(1):58-65.

54. Moore G, Redman S, Butow P, Haynes A. Deconstructing knowledge brokering for commissioned rapid reviews: an observational study. Health Res Policy Syst. 2018;16(1):120.

55. Elledge C, Avworo A, Cochetti J, Carvalho C, Grota P. Characteristics of facilitators in knowledge translation: An integrative review. Collegian. 2019;26(1):171-82.

56. Booth A. Bridging the 'Know-do gap': a role for health information professionals? Health Info Libr J. 2011;28(4):331-4. 
57. Glegg SM, Hoens A. Role Domains of Knowledge Brokering: A Model for the Health Care Setting. J Neurol Phys Ther. 2016;40(2):115-23.

58. Russell DJ, Rivard LM, Walter SD, et al. Using knowledge brokers to facilitate the uptake of pediatric measurement tools into clinical practice: a before-after intervention study. Implement Sci. 2010;5:92.

59. Roxborough L, Rivard L, Russell D. Knowledge Brokering in Health Care. CanChildCentre for Childhood Disability Research. Fact Sheet: Keeping Current; 2010.

60. Lomas J. The in-between world of knowledge brokering. BMJ. 2007;129-132.

61. Metzler MJ, Metz GA. Analyzing the barriers and supports of knowledge translation using the PEO model. Can J Occup Ther. 2010;77(3):151-8.

62. King G, Currie M, Smith L, Servais $M$, McDougall J. A framework of operating models for interdisciplinary research programs in clinical service organizations. Eval Program Plann. 2008;31(2):160-73.

63. Hobbs FR, Taylor CJ. Academic primary care: at a tipping point? Br J Gen Pract. 2014;64(622):2145.

64. Thompson GN, Estabrooks CA, Degner LF. Clarifying the concepts in knowledge transfer: a literature review. J Adv Nurs. 2006;53(6):691-701.

65. Van Kammen J, de Savigny D, Sewankambo N. Using knowledge brokering to promote evidencebased policy-making: The need for support structures. Bull World Health Organ. 2006;84(8):608-12.

66. Kislov R, Wilson P, Boaden R. The 'dark side' of knowledge brokering. J Health Serv Res Policy. 2017;22(2):107-12.

67. Lavis JN, Permanand G, Team BS. A way to approach knowledge brokering: the BRIDGE framework and criteria. In: Bridging the worlds of research and policy in European health systems [Internet]. European Observatory on Health Systems and Policies; 2013.

68. Knight C, Lightowler C. Reflections of'knowledge exchange professionals' in the social sciences: emerging opportunities and challenges for university-based knowledge brokers. Evidence policy: a journal of research debate practice. 2010;6(4):543-56.

69. Valente TW, Pumpuang P. Identifying opinion leaders to promote behavior change. Health Educ Behav. 2007;34(6):881-96.

70. Kang H. The prevention and handling of the missing data. Korean J Anesthesiol. 2013;64(5):402-6.

71. Cochrane LJ, Olson CA, Murray S, Dupuis M, Tooman T, Hayes S. Gaps between knowing and doing: understanding and assessing the barriers to optimal health care. J Contin Educ Health Prof. 2007;27(2):94-102.

72. Rivard LM, Russell DJ, Roxborough L, Ketelaar M, Bartlett DJ, Rosenbaum P. Promoting the use of measurement tools in practice: a mixed-methods study of the activities and experiences of physical therapist knowledge brokers. Phys Ther. 2010;90(11):1580-90.

73. Dogherty EJ, Harrison MB, Graham ID. Facilitation as a role and process in achieving evidence-based practice in nursing: a focused review of concept and meaning. Worldviews Evid Based Nurs. 
2010;7(2):76-89.

74. Janes N, Fox M, Lowe M, McGilton K, Schindel-Martin L. Facilitating best practice in aged care: exploring influential factors through critical incident technique. Int $\mathrm{J}$ Older People Nurs. 2009;4(3):166-76.

75. Mallidou AA, Atherton P, Chan L, Frisch N, Glegg S, Scarrow G. Core knowledge translation competencies: a scoping review. BMC Health Serv Res. 2018;18(1):502.

76. Conklin J, Lusk E, Harris M, Stolee P. Knowledge brokers in a knowledge network: the case of Seniors Health Research Transfer Network knowledge brokers. Implement Sci. 2013;8:7.

77. Hammami H, Amara N, Landry R. Organizational climate and its influence on brokers' knowledge transfer activities: A structural equation modeling. Int J Inf Manage. 2013;33(1):105-18.

78. Long JC, Cunningham FC, Braithwaite J. Bridges, brokers and boundary spanners in collaborative networks: a systematic review. BMC Health Serv Res. 2013;13:158.

79. Dagenais C, Laurendeau MC, Briand-Lamarche M. Knowledge brokering in public health: A critical analysis of the results of a qualitative evaluation. Eval Program Plann. 2015;53:10-7.

80. Dobbins M, Robeson P, Ciliska D, et al. A description of a knowledge broker role implemented as part of a randomized controlled trial evaluating three knowledge translation strategies. Implement Sci. 2009;4:23.

81. Robeson P, Dobbins M, DeCorby K. Life as a knowledge broker in public health. Journal of the Canadian Health Libraries Association/Journal de l'Association des bibliothèques de la santé du Canada. 2008;29(3):79-82.

82. Bornbaum CC, Kornas K, Peirson L, Rosella LC. Exploring the function and effectiveness of knowledge brokers as facilitators of knowledge translation in health-related settings: a systematic review and thematic analysis [published correction appears in Implement Sci. 2015;10:171]. Implement Sci. 2015;10:162.

\section{Figures}




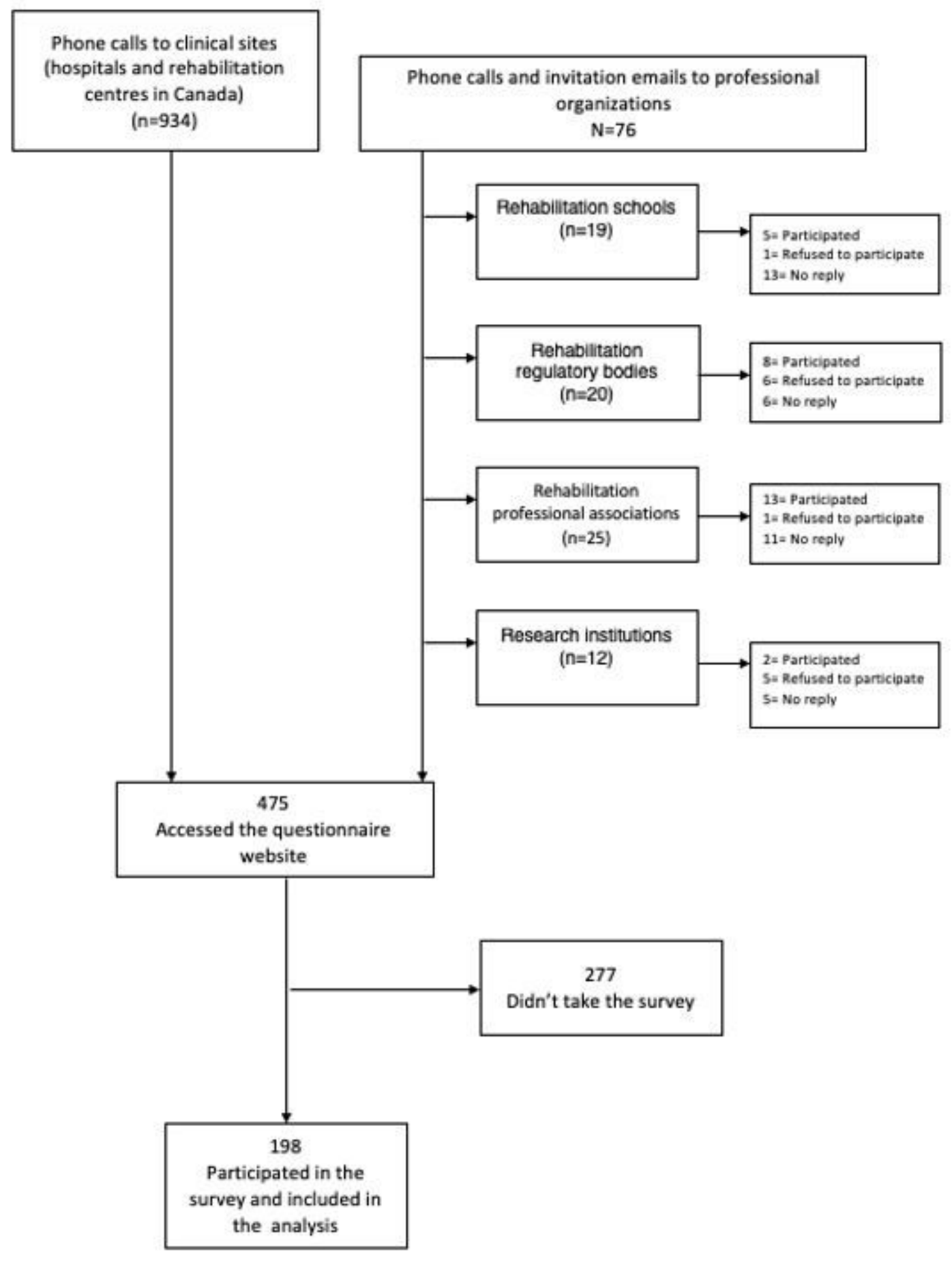

Figure 1

Flowchart of the recruitment strategies 


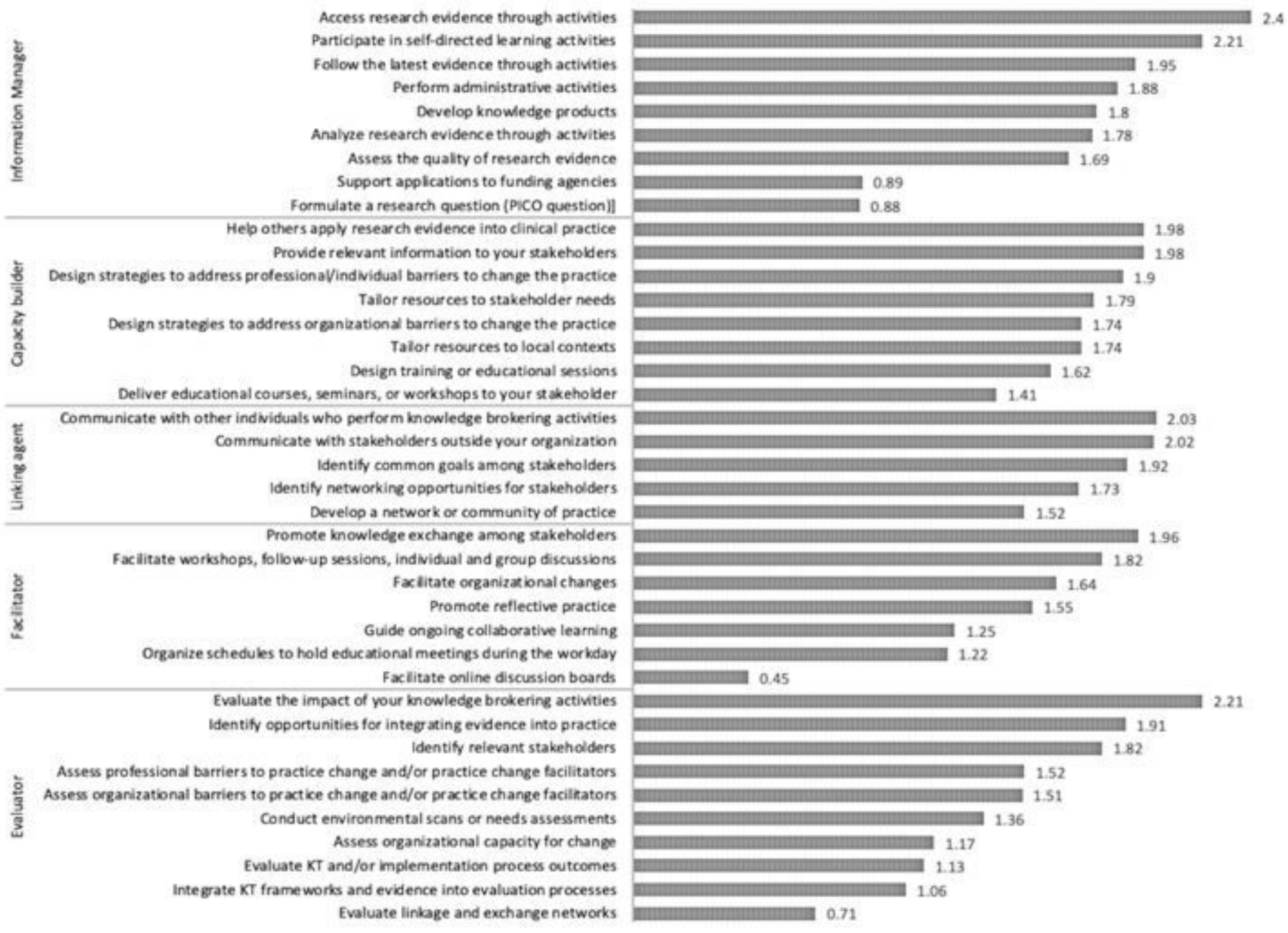

Figure 2

The weighted-average of performing each task corresponding to each knowledge brokering role across all participants

\section{Supplementary Files}

This is a list of supplementary files associated with this preprint. Click to download.

- Additionalfile4.docx

- Additionalfile3.docx

- Additionalfile2.docx

- Additionalfile1.docx 